\title{
Proceso de Certificación de Buenas Prácticas Agrícolas (BPA) Según Norma ICA 30021 , en cultivo de aguacate Hass (Persea americana Mill), municipio de Isnos - Departamento del Huila
}

Process of Certification of Good Agricultural Practices (BPA) According to ICA 30021, in culture of Hass avocado (Persea americana Mill), municipality of Isnos, Huila

\section{Nelly María Méndez Pedroza ${ }^{1}$}

Ing. Forestal, Doctora en Desarrollo Sostenible

Escuela de Ciencias Agrícolas, Pecuarias y del Medio Ambiente -ECAPMA.

Universidad Nacional Abierta y a Distancia -UNAD.

https://orcid.org/0000-0003-4219-0378

nelly.mendez@unad.edu.co

\section{Gloria Patricia Murcia Muñoz ${ }^{2}$}

Estudiante Agronomía

Escuela de Ciencias Agrícolas, Pecuarias y del Medio Ambiente -ECAPMA. Universidad Nacional Abierta y a Distancia -UNAD.

glopamurcia@hotmail.com

\section{Leonardo Rincón Ramírez ${ }^{3}$}

Estudiante Agronomía

Escuela de Ciencias Agrícolas, Pecuarias y del Medio Ambiente -ECAPMA. Universidad Nacional Abierta y a Distancia -UNAD. leonardo110rvspec@hotmail.com

\section{RESUMEN}

El presente documento se realizó con el objetivo de realizar la trazabilidad en la realización del proceso de certificación de buenas prácticas agrícolas (BPA); teniendo en cuenta la norma ICA 30021 del 28 de abril de 2017, en el cultivo de aguacate (Persea americana Hass) en la unidad productiva la Floresta del municipio de Isnos del departamento del Huila. Para el desarrollo del documento se tuvieron en cuenta todas las actividades de planeación y dirección del cultivo de aguacate (Persea americana Hass), como también se tuvo presente la identificación de zonas de peligro dentro de la unidad productiva, áreas de instalación y adecuación de infraestructura, también se 
manejó el tema de calidad y tratamiento de aguas, control integrado del cultivo en cuanto a plagas y enfermedades, el bienestar de los trabajadores y la protección ambiental de la unidad productiva, con procesos que son soportados con registros y documentación del Instituto Agropecuario ICA, y otras empresas privadas para análisis tanto de suelo como de agua.

Palabras Clave: Agricultura; certificación; control biológico; desarrollo rural; enfermedades; exportación; manejo de aguas residuales; plagas.

\section{ABSTRACT}

The applied project has been carried out with the objective of carrying out the process of certification of good agricultural practices (GAP); taking into account the ICA 30021 standard of April 28, 2017, in the cultivation of avocado (Persea americana Hass) in the production unit of La Floresta in the municipality of Isnos, department of Huila.

This work has been carried out with different activities taking into account the planning and management of the avocado cultivation (Persea americana Hass), as well as the identification of danger zones within the productive unit, such as installation areas and adaptation of infrastructure. , the issue of quality and water management, integrated management of the crop, integrated management of pests and diseases, as well as the wellbeing of the workers and the environmental protection of the productive unit were also handled, where these processes have been supported by documentation and records of each process, by different entities such as Instituto Agropecuario ICA, and other private companies for soil and water analysis.

Keywords: Agriculture; Certification; Rural development; Exportation; Wastewater management; Pests; Diseases; Biological control.

\section{INTRODUCCIÓN}

En el desarrollo del documento se ha aplicado la normatividad respecto a las buenas prácticas agrícolas (BPA) atendiendo la Norma ICA 30021 
del 28 de abril de 2017, en el cultivo de aguacate (Persea americana Hass), en el predio la Floresta del municipio de Isnos - Departamento del Huila, donde actualmente se encuentra en estado terminado y en funcionamiento la certificación, en que ya se ha emitido Certificación de predio como exportador y registro en buenas prácticas agrícolas para la unidad productiva. El predio ya cuenta con capacidad para generar la actividad productiva sobre el cultivo de aguacate, generando ambientes sanos y sostenibles con el medio ambiente y presentando un mayor beneficio al momento de comercializar las producciones.

Es de enunciar que "Cuando las unidades productivas agrícolas en forma individual o forma conjunta con otros productores están dispuestas a desarrollar e implementar el Sistema de Gestión de Calidad (SGC) y deciden dar cumplimiento a una norma o protocolo de acuerdo a las exigencias de los compradores o al interés en un mercado determinado. Este proceso debe ser inspeccionado y aprobado por una entidad independiente y especializada, que para el caso son las empresas certificadoras (CAPLAM, 2006; Fonseca, et al., 2011). Por tanto, es de resaltar que para este caso la entidad certificadora ha sido el Instituto Colombiano Agropecuario - ICA; quien ha visitado el predio La Floresta del municipio de San Agustín del departamento del Huila, y emitido el proceso de certificación, siendo de gran importancia dado que, el producto a ofrecer tiene las condiciones ideales de inocuidad y es producido bajo las normas ICA, lo que redundará en bienestar, pues el producto tendrá valor agregado aumentando el precio que se verá reflejado en ganancias y mejora de salarios a trabajadores.

\section{METODOLOGÍA}

El diagnostico se realizó por medio de observación y registro de datos llevando a cabo el reconocimiento de cada unidad como la productiva, infraestructura, ambiental, social y las diferentes características que se encontraron en el momento de indagar en el predio La Floresta.

Las Buenas Prácticas Agrícolas son prácticas orientadas a la sostenibilidad ambiental, económica y social para los procesos productivos de la explotación agrícola que garantizan la calidad e inocuidad de los alimentos y de los productos no alimenticios. (ICA, 2017).

El desarrollo del análisis y evaluación del proceso de certificación de buenas prácticas agrícolas en el predio La Floresta con la norma ICA 30021 de 28 de abril del 2017, se llevó a cabo teniendo en cuenta los 
siguientes pasos los cuales se analizaron para un exitoso proceso. 1. Buenas prácticas agrícolas, 2. Planeación en el cultivo de aguacate, 3. Revisión y análisis de los peligros presentados en el predio, 4. Revisión de las áreas construidas, 5. Análisis de la calidad del agua usada en el predio, 6. Manejo integrado del cultivo de aguacate, 7. Manejo integrado de plagas en el cultivo, 8. Protección y bienestar de los trabajadores del predio, 9. Manejo y protección del ambiente, 10. Documentación registros para llevar la trazabilidad de todos los procesos realizados en el predio y en el cultivo del aguacate.

En la figura 1 se muestra el proceso en forma gráfica, resumiendo los puntos anotados anteriormente.

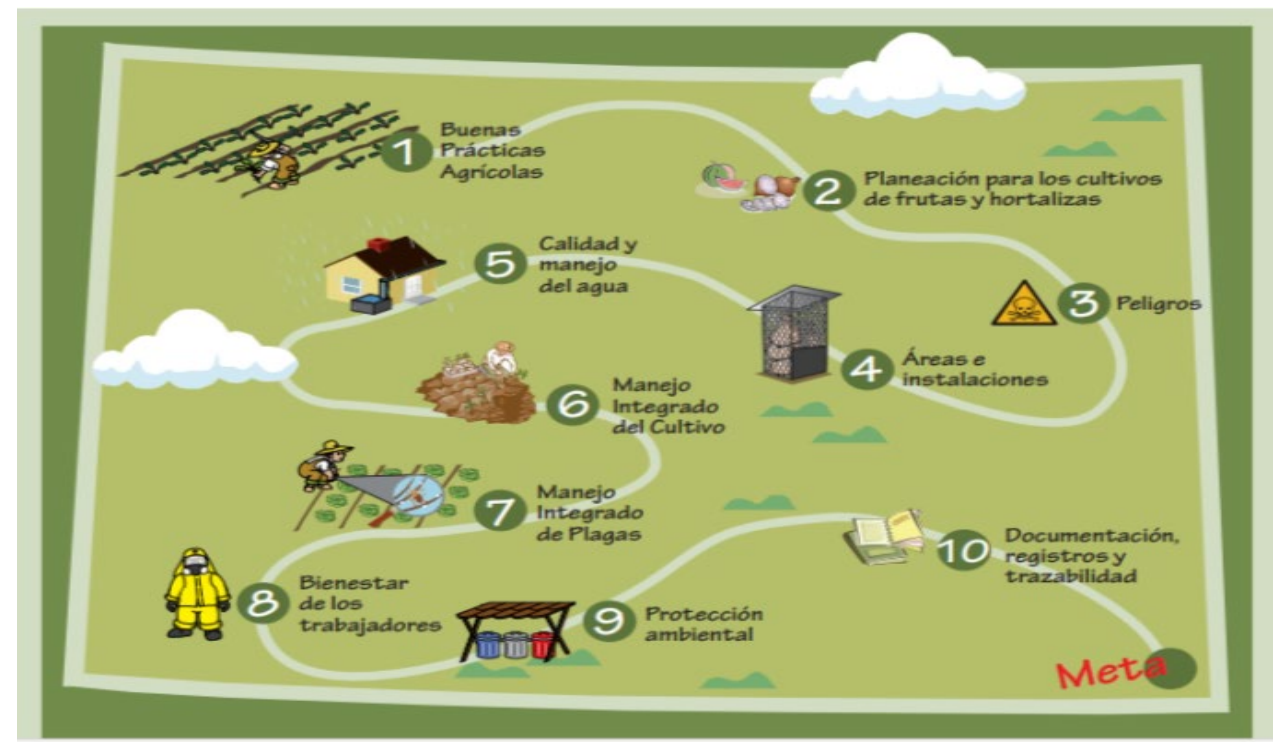

Figura 1. Proceso de certificación de Buenas Prácticas Agrícolas.

Fuente: Instituto Colombiano Agropecuario - ICA (2017)

Dando alcance a los pasos anteriores, se logrará con la certificación lo siguiente:

El mejoramiento del proceso de BPA en el cultivo de aguacate Hass Identificación, análisis y mejoramiento de los peligros dentro de la unidad productiva respecto de riesgos biológicos, físicos, químicos y entre otros determinando los niveles de estos como altos, medios y bajos.

Contribución al mejoramiento de áreas e instalaciones con la identificación de sectores dentro de la unidad productiva.

Mejorar la calidad y manejo del agua con el seguimiento oportuno dentro de la unidad productiva. Si se sabe mantener la calidad y cantidad del agua en la unidad productiva, protegemos el ambiente y contribuimos a producir el producto agrícola. 
Manejo integrado del cultivo y protección del mismo al ataque de plagas y enfermedades teniendo presente:

a. Manejo de suelos

b. Material de propagación.

c. Nutrición de plantas

d. Protección del cultivo.

La recolección de información, almacenamiento y análisis, se realizó tomando datos de los procesos que se realizaron en la unidad productiva La Floresta y se almacenaron en base de datos con el programa Excel donde se realizaron procesos de análisis y graficación de datos para los resultados definitivos del proceso de certificación. (Cordova, 2007).

Para el proceso de certificación en Buenas Prácticas Agrícolas (BPA) debe estar apoyado por la implementación del Sistema de Gestión de la Calidad (SGC) que como dice Fonseca C, Muñoz P, \& Cleves L. (2011), requiere de la construcción y puesta en funcionamiento de documentos o manuales soporte en aspectos administrativos, productivos y de organización de productores, que están estructurados para responder a las exigencias de una norma, protocolo o código de conducta.

\section{RESULTADOS}

Proceso de certificación de las buenas prácticas agrícolas Según la norma ICA 30021 en la unidad productiva La Floresta del municipio de Isnos.

\section{Diagnóstico de la unidad productiva La Floresta, Municipio de Isnos}

El diagnóstico realizado sobre el estado de la unidad productiva al iniciar el proceso de certificación no contaba con esta o alguna que soportara sus actividades económicas. Los faltantes para lograr la certificación entre otros se encuentra, el registro de predio, certificaciones en BPA, certificación como exportador, adecuaciones del sistema de drenaje de aguas, adecuaciones en señalizaciones, edificaciones para bodegas adecuadas, análisis físico químico de aguas y análisis de suelos. La figura 2 muestra el estado del predio antes de iniciar el proceso de certificación en Buenas Prácticas Agrícolas. 
Periodo Inicial de los procesos de certificacion y adecuacion del predio la Floresta 05/05/2017

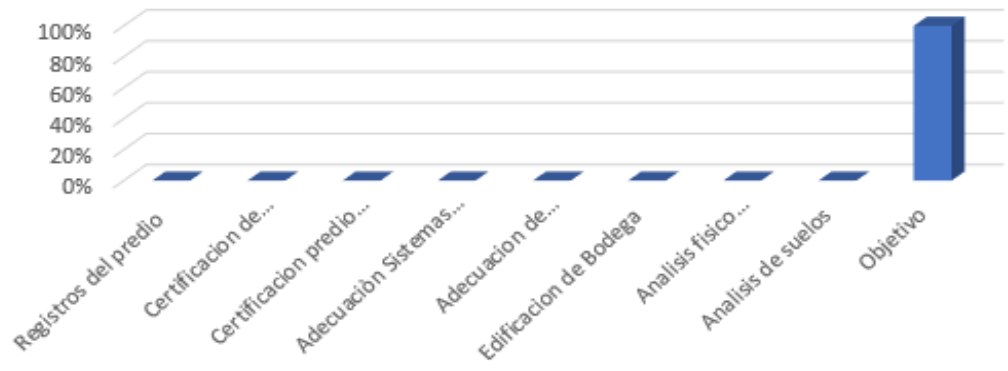

Figura 2. Periodo inicial del proceso de certificación y adecuación del predio la Floresta-mayo de 2017

Luego de aplicados los criterios de buenas prácticas agrícolas establecidos en la norma ICA 30021 de 28 de abril del 2017, dentro de la unidad productiva La Floresta en el municipio de Isnos, se registra el siguiente cumplimiento que permitió la obtención de la certificación en el manejo de la sanidad, cumpliendo acciones y disposiciones necesarias para la prevención, erradicación, o el manejo de enfermedades, plagas, malezas o cualquier otro organismo dañino que afecten las plantas y sus productos, actuando en permanente armonía con la protección y preservación de los recursos naturales. (Diario Oficial de Colombia, 2017). Esto se puede constrastar con los trabajos de LópezMeneses et al. (2016).

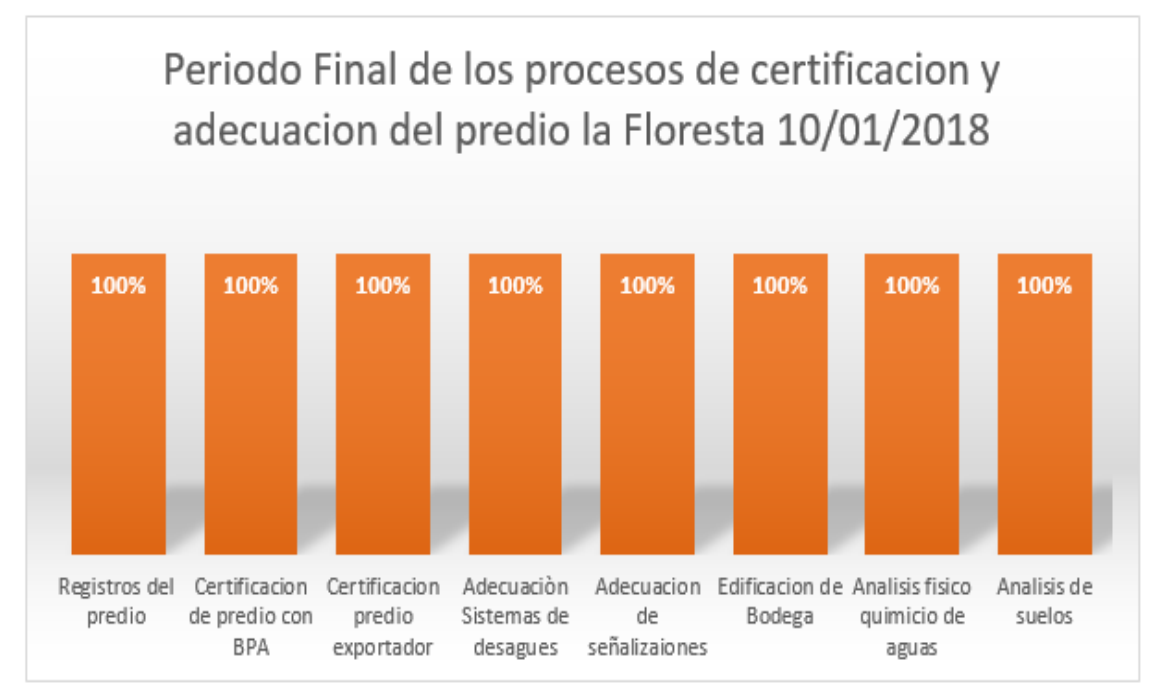

Figura 3. Periodo Final de los procesos de certificación y adecuación del predio la Floresta-enero de 2018 
Seguimiento de actividades de adecuación y certificación de Unidad Productiva.

\section{Construcción Bodega en unidad productiva la Floresta.}

Se mejoraron y establecieron varias construcciones entre ellas, la bodega de almacenamiento, definiéndose las medidas convenientes para la necesidad de almacenamiento de insumos agrícolas del predio, lo mismo que organización del sistema de agua, teniendo en cuenta las proyecciones para la bodega permitiendo dar viabilidad a la certificación del predio, lo que asegura mejor ambiente de trabajo y control de inventario de herramientas.

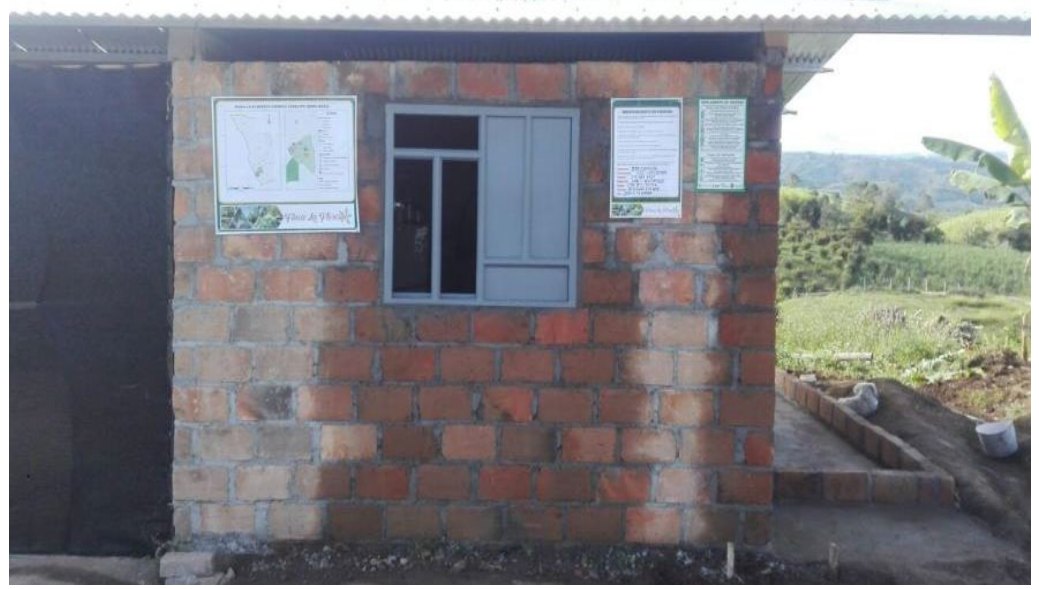

Figura 4. Bodega construida de la unidad productiva la Floresta
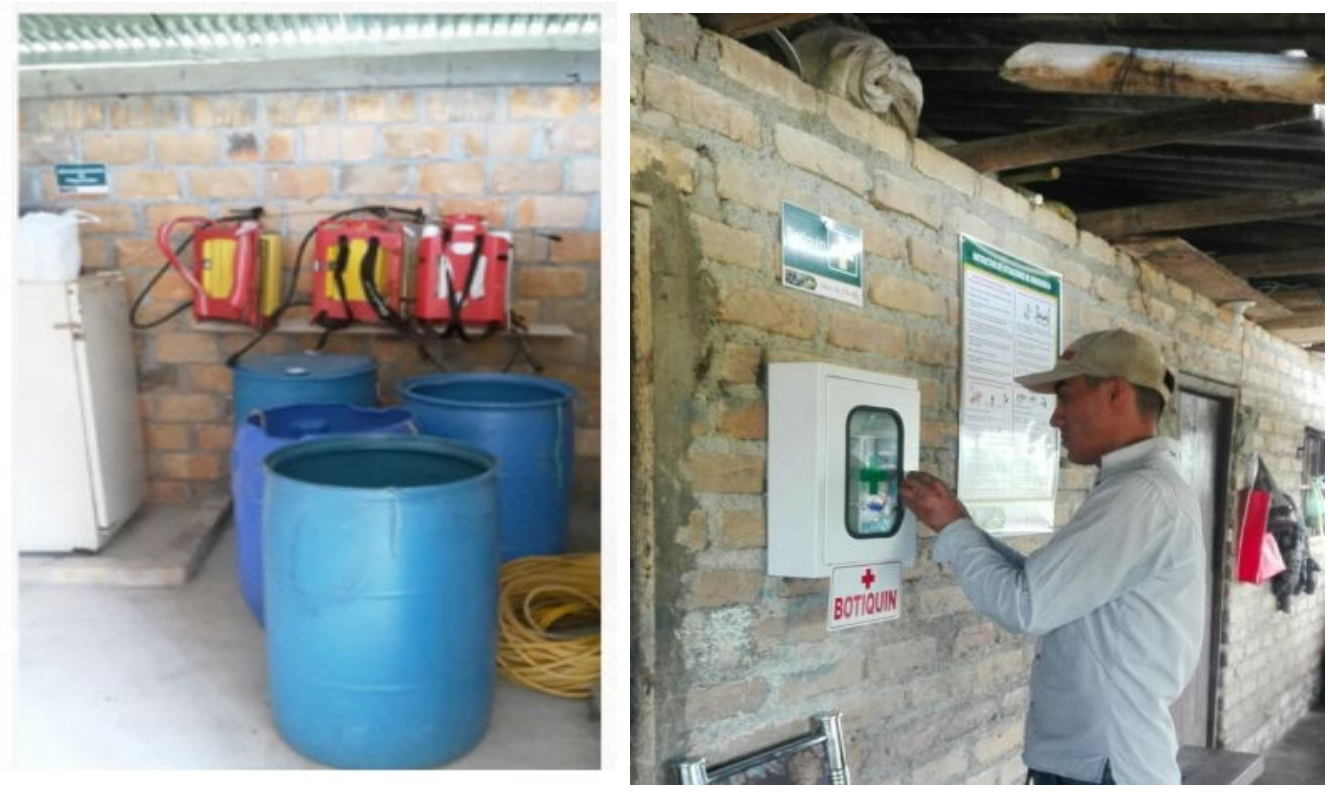

Figura 5. Disposición y adecuación de implementos e insumos en la bodega de almacenamiento 
Se observan herramientas e implementos para trabajo disponibles en bodega, como bomba manual para fumigar, manguera de presión para estacionaria y timbos para el depósito de agua. La instalación de bodega se encuentra terminada en un $100 \%$ y lista para la utilización, la cual consta de techo, ventanas y descripciones de uso. También se adecua y realiza la instalación del botiquín para primeros auxilios, que será utilizado en caso de accidentes dentro de unidad productiva, los cuales deben ser atendidos de forma oportuna y puntual.

Señalización de seguridad para casos de intoxicaciones en el personal manipulador. Se establece la señalización en un lugar visible y de acuerdo a la normatividad existente para tal fin.

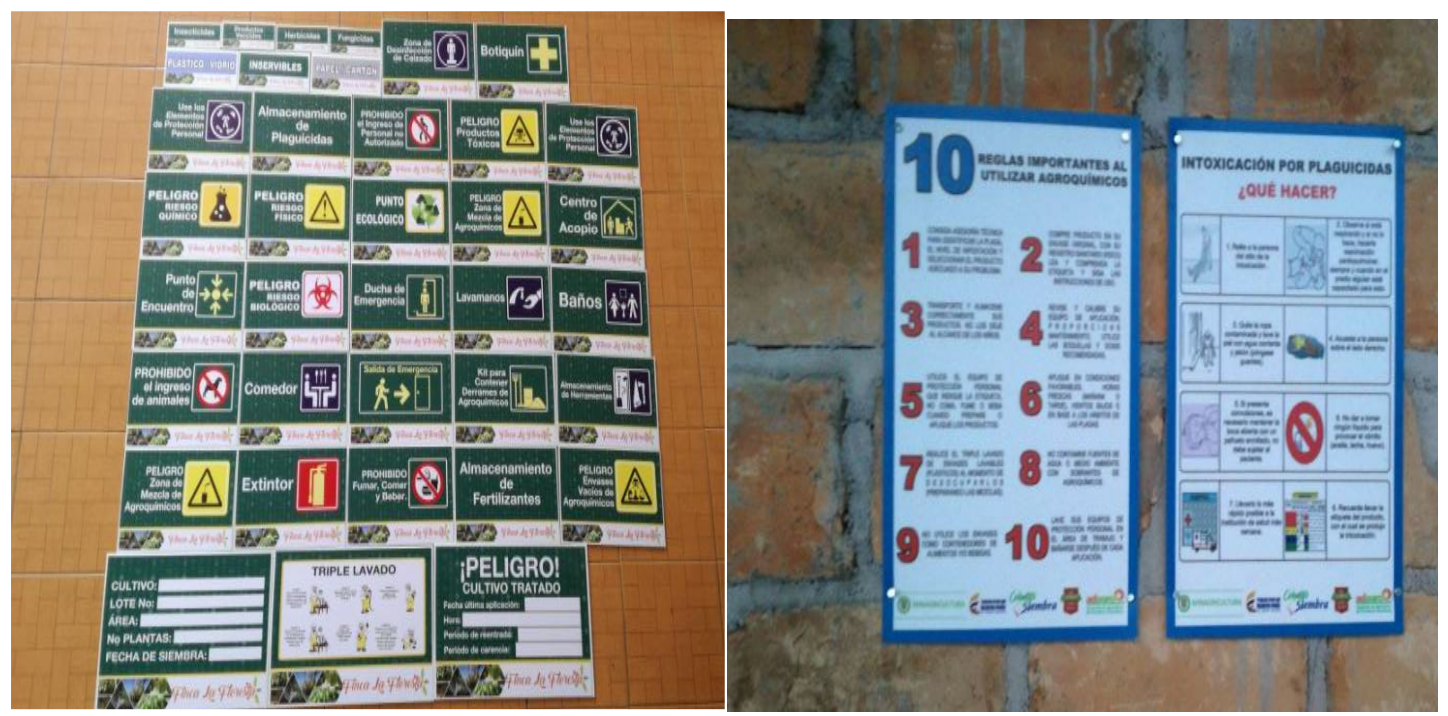

Figura 6. Disposición de señales en el predio de acuerdo a normatividad existente

La señalización protege al productor y trabajadores de incidentes relacionados con la salud, calidad e inocuidad y el incumplimiento de los parámetros establecidos en la comercialización y producción para cumplir los requerimientos de las buenas prácticas de manufactura. (CCI, 2017).

\section{Croquis de la unidad productiva la Floresta.}

Dando cumplimiento a una de las exigencias de la Resolución 30021 sobre los requisitos para la Certificación en Buenas Prácticas Agrícolas en producción primaria de vegetales y otras especies para consumo 
humano., se realiza el croquis de llegada y plano del predio, indicando las áreas destinadas al cultivo, como se muestra en la siguiente figura.

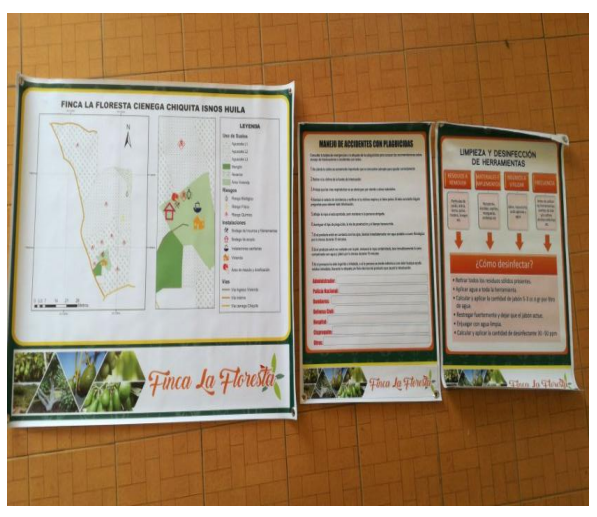

Figura 7. Croquis del predio La Floresta

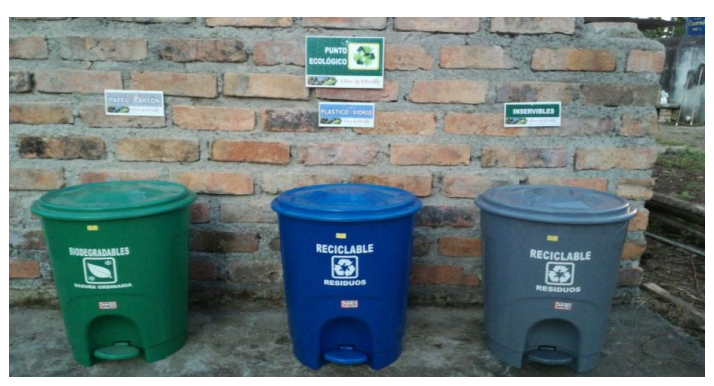

Figura 8. Disposición de recipientes para el reciclaje

La ubicación del sitio de reciclaje es fundamental para dar buen uso a los materiales de desecho que se generen en la vivienda y predio con el ánimo de proteger y conservar el medio ambiente. En la imagen se observan los contenedores para la disposición del vidrio, papel y residuos inorgánicos.

\section{Adecuación de sistema de drenaje de aguas}

Sistema de drenaje de aguas, permitiendo el adecuado flujo de aguas en la infraestructura de predio. Según la resolución, este sistema debe estar dotado y en condiciones óptimas de limpieza y construidos con materiales fáciles de limpiar y con sistema de evacuación de aguas servidas diseñadas para prevenir la contaminación en el campo y estar a una distancia no menor de 100 metros de fuentes hídricas y a 15 metros de donde se manipulen o se almacenen productos agrícolas. La siguiente figura muestra lo construido en el predio. 


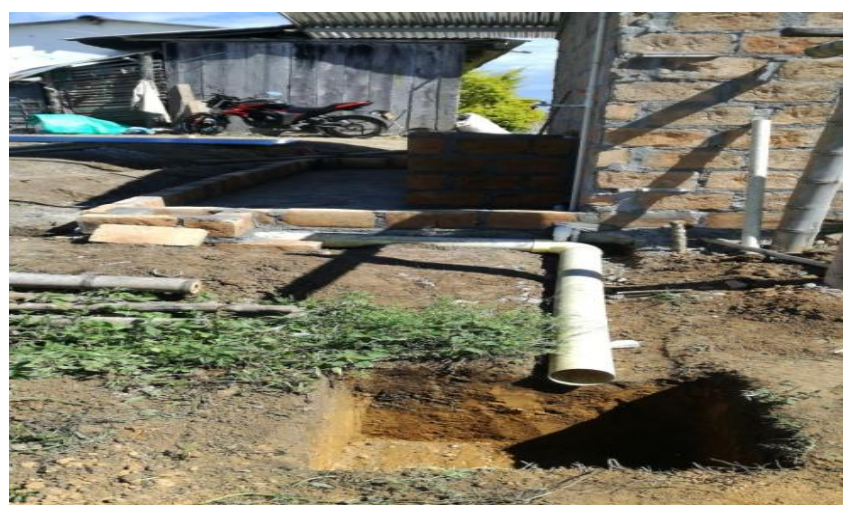

Figura 9. Adecuación de sistema de drenaje de aguas

La resolución manifiesta que toda persona natural o jurídica que posean a cualquier título cultivos de vegetales y otras especies para consumo humano y deseen certificar el predio productor en buenas prácticas agrícolas, debe realizar solicitud anexando documentos de representación legal. asistente técnico del predio, croquis o plano del predio y análisis de agua entre otros.

\section{Análisis Físico químico del agua de la unidad productiva la Floresta}

Respecto al análisis Físico químico del agua de la unidad productiva La Floresta, que es una de las condiciones pedidas por el ICA para certificación de buenas prácticas, en la figura 11 se muestra el análisis del agua usada en el predio. 


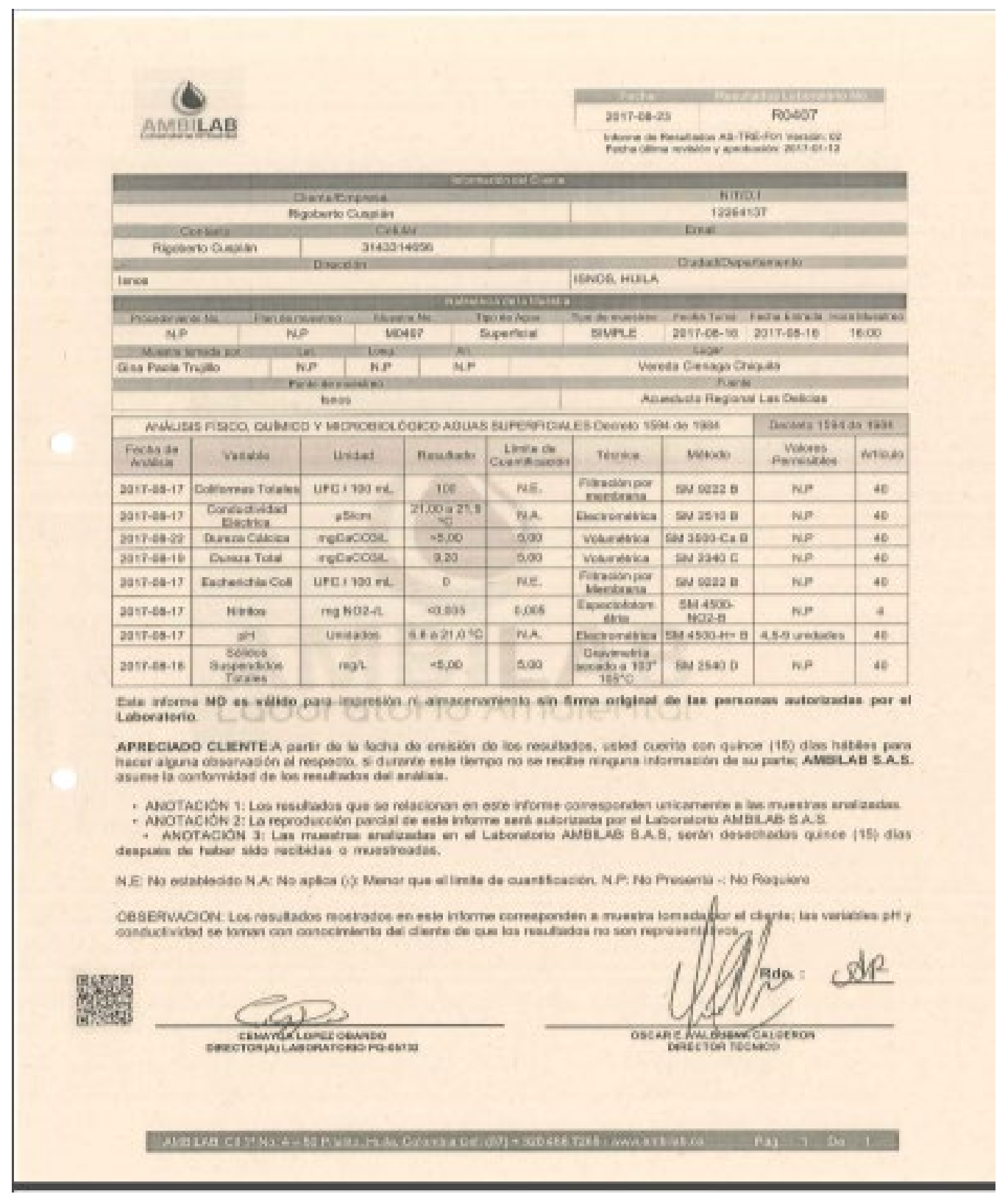

Figura 11. Análisis Físico químico del agua de la unidad productiva la Floresta 


\section{Análisis de suelos de la unidad productiva La Floresta}

Se realiza el análisis de suelos de la unidad productiva La Floresta, como actividad complementaria para obtener la certificación en buenas prácticas.

Para dar cumplimiento a otra de las exigencias de la normatividad de buenas prácticas, es necesario presentar un análisis de realizado por un agrónomo o ingeniero agrónomo respecto del estado de los suelos donde se encuentra el cultivo y las recomendaciones realizadas por el mismo para fertilización y manejo del cultivo.

Interpretación y recomendaciones del análisis de suelos le han permitido al agricultor programar y realizar fertilizaciones y manejo integral del cultivo.

\section{Certificado del Instituto Colombiano - ICA de Buenas prácticas agrícolas del predio la Floresta.}

Luego de surtido todo el procedimiento demandado por la normatividad respecto de las certificaciones en Buenas Prácticas Agrícolas, se obtuvo el mismo por una vigencia de dos años y para la renovación se realizará previa solicitud al ICA por parte del productor.

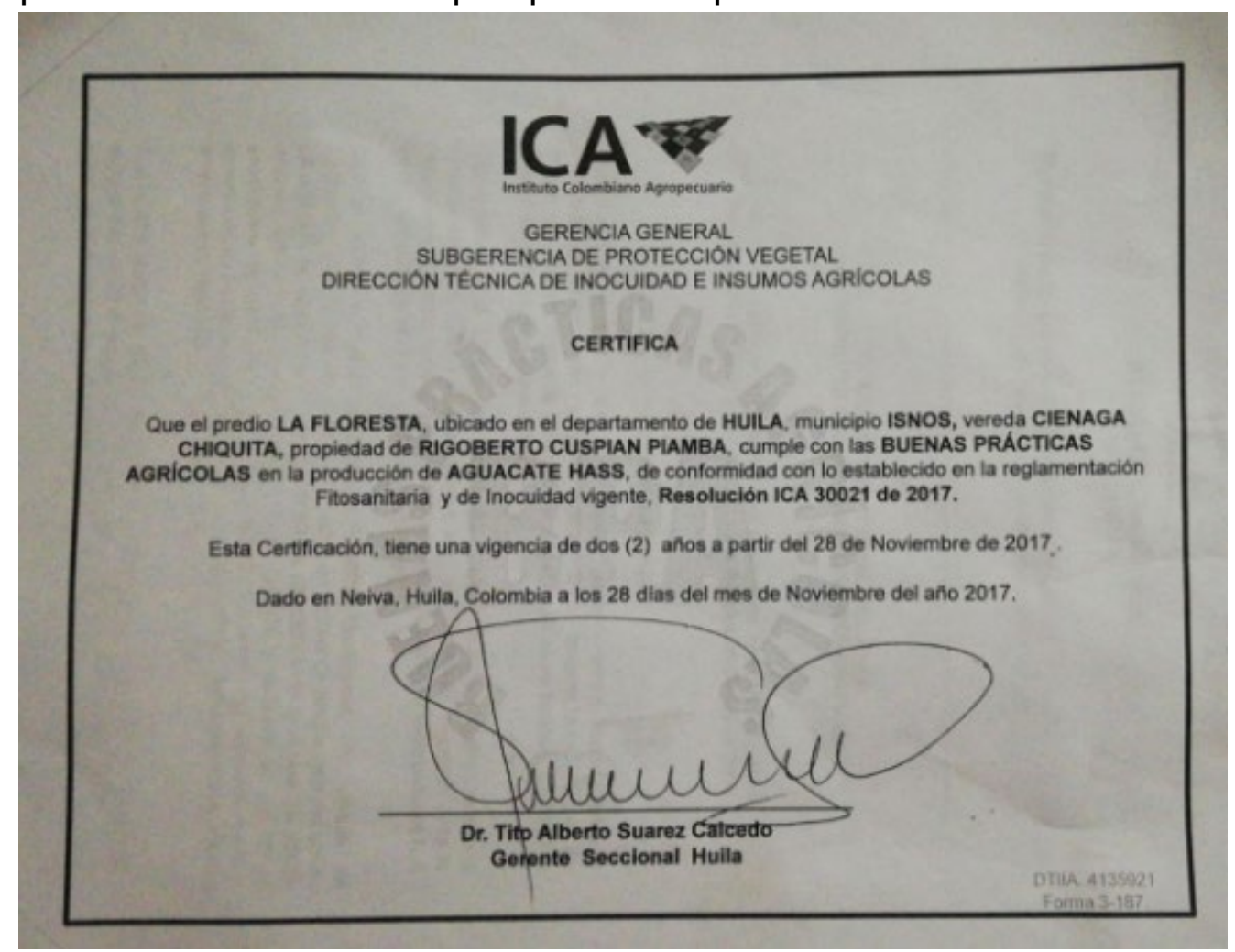

Figura 14. Certificado otorgado por el ICA en Buenas Prácticas Agrícolas. Fuente: Instituto Colombiano Agropecuario - ICA. 
En este aspecto es de resaltar que la certificación se logró gracias al trabajo mancomunado entre productor - estudiantes y siguiendo adecuadamente la normatividad a pesar de los vacíos de la misma, que como manifiesta (Toro y Martínez, 2013; Montes y Sánchez, 2016; ) los métodos para evaluar impactos ambientales en el proceso de Licenciamiento Ambiental en Colombia, se caracterizan por un alto nivel de incertidumbres y sesgo, disminuyéndose la efectividad del proceso.

\section{CONCLUSIONES Y RECOMENDACIONES}

El proyecto aplicado se ha realizado con el objetivo de analizar, evaluar y ejecutar el proceso de certificación de buenas prácticas agrícolas (BPA) según norma ICA 30021 de 28 de abril del 2017, en el cultivo de aguacate (Persea americana Hass), predio la Floresta del municipio de Isnos - Departamento del Huila, en compañía del agricultor, lográndose la certificación del predio.

El proyecto realizado da fe de que, si el productor es metódico y sigue correctamente la guía del Ica, si es factible la certificación de la unidad productiva bajo la norma ICA 30021 de 28 de abril del 2017, para contribuir a la calidad y el valor económico del producto que en este caso es el aguacate Has, permitiendo que el productor genere valor agregado a su producción y por ende a mejorar la calidad de vida. Además de conservar y proteger los recursos naturales (Corredor, Camargo y Fonseca, 2012; Vinasco, 2017; Zapata y Llano, 2014).

Es de tener presente que en el proyecto ha definido los diferentes puntos para llegar a la certificación de buenas prácticas agrícolas y ser categorizado como predio exportador, esto partiendo desde la planeación del cultivo de aguacate (Persea americana Hass), permitiendo reconocer las diferentes áreas para la unidad productiva desde áreas de Peligro para personal, áreas de instalaciones de bodega e insumos.

Para la certificación se tuvo en cuenta temas de calidad y manejo de aguas dentro del predio con análisis fisicoquímicos se han conocido características ideales para el manejo del recurso hídrico.

Como otro tema de importancia para el productor llevado a cabo fue el manejo integrado de plagas y enfermedades siguiendo técnicas de reconocimiento como identificar índice de plagas por medio de muestras en la selección de árboles de aguacate observando el estado fitosanitario y esto permitiendo sacar porcentajes de infestaciones 
dentro del cultivo para descartar cualquier patógeno que afecte la calidad del producto al momento de ser comercializado. (Experian, 2018).

También se tuvo en cuenta aspectos que debía reconocer el productor para el bienestar de los trabajadores, permitiendo llevar un adecuado uso de herramientas y materiales e insumos para el cultivo sin afectar la integridad de las personas que intervienen en el cultivo.

La certificación en buenas prácticas agrícolas ha permitido que el productor obtenga las características ideales para producir de forma de sosteniblemente con el medio ambiente sin degradas los recursos naturales suelo, agua, fauna, flora y permitiendo entender la importancia de estos hacia un futuro sosteniblemente.

El proceso de certificación de la unidad productiva tuvo que pasar por el desarrollo de actividades como el registro del predio como exportador y tramitar con el instituto agropecuario ICA, la certificación en buenas prácticas agrícolas donde tuvo lugar la visita del personal encargado de esta entidad para verificar el estado de la unidad productiva para proceder con los trámites legales.

Se tuvo presentes actividades de importancia para esta certificación como la adecuación de sistema de drenajes de aguas, adecuar señalizaciones dentro del predio, adecuación de bodega de insumos agrícolas y herramientas, como los soportes de análisis físico químico de aguas y análisis de suelos ya mencionados anteriormente.

Todos estos procesos fueron llevados a cabo por el productor quien es el ejecutor y los estudiantes del programa de agronomía de la Universidad Nacional Abierta y a Distancia - UNAD, quienes han permitido guiar al productor y también personal externo como asesores del instituto agropecuario ICA, entre otras entidades privadas como las que realizaron y análisis de suelos y agua.

Por lo tanto, se tiene que para este tipo de procesos de certificación es recomendable proyectar actividades e ir anticipando temas como la inocuidad del cultivo por parte de los productores que requieran generar este proceso y que para cuando se esté ejecutando el proyecto permitan agilidad en la certificación.

Aunado a esto, es necesario considerar la biotecnología aplicada al sector agroindustrial, que es la que presenta un mayor desarrollo en Colombia en comparación a otros subsectores, podría visualizarse de forma positiva el futuro de la aplicación sustentable de la biotecnología agropecuaria enmarcada dentro de las tendencias mundiales a los cultivares de aguacate, no obstante se requiere de esfuerzos significativos 
en investigación básica y aplicada que coloquen a Colombia a la vanguardia del desarrollo biotecnológico agropecuario (Montenegro Gómez \& Hernández Ossa, 2015). Es importante en este punto tener en cuenta los trabajos de Gamboa (2015), Giraldo y Valencia (2010), Vinasco (2017), Bonilla y Caetano (2013), Matos de Oliveira (2014), Mendoza, Cano y Rojas (2015), que consideran el desarrollo del país y de las comunidades desde otras perspectivas.

En temas social y medioambiental, es de gran aporte la certificación de predios permitiendo producir sosteniblemente con los recursos naturales fauna, flora, suelo, agua, llevando el bueno manejo de basuras y residuos sólidos que pueda generar la actividad económica.

\section{BIBLIOGRAFÍA}

Bonilla Morales, M., \& Caetano, C. (2013). Inventario y valoración de la flora utilizada por la vereda Santa Teresa, Palmira (Valle del Cauca). Revista de Investigación Agraria y Ambiental, 4(1), 8999. doi:https://doi.org/10.22490/21456453.985

CCI, ICA. (2017). Manual temático del facilitador en BPA. Obtenido de https://www.ica.gov.co/Areas/Agricola/Servicios/InocuidadAgricola/Capacitacion/anexosManual.aspx

Córdoba, J. E. (9 de 11 de 2007). Cap. 10 Técnicas e instrumentos de recolección de datos. Recuperado el 20 de Febrero de 2010, de Elaboración de Proyectos de Investigación: http://www.mailxmail.com/curso-elaboracion-proyectosinvestigacion/tecnicas-instrumentos-recoleccion-datos

Corredor Camargo, E., Fonseca Carreño, J., \& Páez Barón, E. (2012). Los servicios ecosistémicos de regulación: tendencias e impacto en el bienestar humano. Revista de Investigación Agraria y Ambiental, 3(1), 77-83. doi:https://doi.org/10.22490/21456453.936 (corredor, Fonseca y Páez, 2012)

Diario Oficial de Colombia. (5 de mayo de 2017). Legislación . (I. C. Agropecuario, Ed.) Obtenido de https://diariooficial.vlex.com.co/vid/resolucion-numero-030021-2017678721361 
Experian; 2018; Control de plagas y enfermedades, recuperado de; http://es.hesperian.org/hhg/A_Community_Guide_to_Environm ental_Health:Control_de_plagas_y_enfermedades_de_las_plant as

ICA, 2018; Medidas de Prevención y manejo cultivo Aguacate Hass Mill; recuperado de; https://www.ica.gov.co/getattachment/4b5b9b6f-ecfc-46e1b9ca-b35cc1cefee2/-nbsp;Manejo-fitosanitario-del-cultivo-deAguacate.aspx

Fonseca C, J. A., Muñoz P, N. A., \& Cleves L, J. A. (2011). El sistema de gestión de calidad: elemento para la competitividad y la sostenibilidad de la producción agropecuaria colombiana.

Revista de Investigación Agraria y Ambiental, 2(1). Obtenido de http://hemeroteca.unad.edu.co/index.php/riaa/article/view/909

Giraldo Díaz, R., \& Valencia T., F. (2010). Evaluación de la sustentabilidad ambiental de tres sistemas de producción agropecuarios, en el corregimiento Bolo San Isidro, Palmira (Valle del Cauca). Revista de Investigación Agraria y Ambiental, 1(2), 7-17. doi:https://doi.org/10.22490/21456453.900

Gómez, M. M. (2006). Introducción a la Metodología de la Investigación Científica. (E. Brujas, Ed.) Recuperado el 20 de Febrero de 2010, de Google Books:

http://books.google.com/books?id=9UDXPe4U7aMC\&pg=PA93\& Ipg $=$ PA93\&dq $=\%$ E2\%80\%9CUn\%2Bexperimento $\% 2 B d e b e \% 2 B$ buscar\%2Bante\%2Btodo\%2Bvalidez\%2Binterna;\%2Bes\%2Bde $\mathrm{cir}, \&$ source $=$ bl\&ots $=b 5 \mathrm{pKKWILAO \& sig=9dZGgUUBddENiopJjBe3}$ G-N-LCA\&hl=en\&ei $=$ AsWAS6WoEcSXtgeDXJX-Bg\&sa $=X \& o i=b$

ICA. (5 de mayo de 2017). RESOLUCIÓN 30021 DE 2017. Obtenido de https://www.icbf.gov.co/cargues/avance/docs/resolucion_ica_3 0021_2017.htm

ICA; 2018; Cultivo de aguacate presea americana Mill, recuperado de; https://www.ica.gov.co/getattachment/4b5b9b6f-ecfc- 
46e1-b9ca-b35cc1cefee2/-nbsp;Manejo-fitosanitario-delcultivo-de-Aguacate.aspx

López-Meneses, C. E., Nieto-Gómez, L. E., Sánchez-Jiménez, W., Posada-Molina, V., Ramírez-Galvis, M. A. (2015). Paros agrarios y negociación entre el gobierno colombiano y la Cumbre Agraria Étnica Campesina y Popular (CACEP). Revista Criterio Libre Jurídico, 12(2), 161-175

http://dx.doi.org/10.18041/crilibjur.2015.v12n2.24209

Martinez M., M. (s.f.). Validez y Confiabilidad en la Metodología Cualitativa. Recuperado el 20 de Febrero de 2010, de Página del Investigador Miguel Martínez: http://miguelmartinezm.atspace.com/Validez\%20y\%20Confiab \%20en\%20la\%20Metod\%20Cualit.htm

Matos de Oliveira, A. (2014). Crítica ecológica al concepto de Desarrollo y nuevas alternativas desde América Latina. Revista de Investigación Agraria y Ambiental, 5(2), 41 - 53. doi:https://doi.org/10.22490/21456453.1325

Mendoza Velásquez, S., Cano Muñoz, J., \& Rojas Sánchez, F. (2015). Acción comunitaria frente al fenómeno del cambio climático, en el páramo de la región del Guavio, Cundinamarca, Colombia. Revista de Investigación Agraria y Ambiental, 6(1), 265 - 279. doi:https://doi.org/10.22490/21456453.1286

Montenegro Gómez, S. P., \& Hernández Ossa, Y. K. (2015). Biotecnología aplicada al desarrollo agropecuario colombiano. Revista de Investigación Agraria y Ambiental, 6(2), 1 - 12. Obtenido de http://hemeroteca.unad.edu.co/index.php/riaa/article/view/140 $8 / 1732$

Montenegro Gómez, S. P., Barrera Berdugo, S. E., \& Valencia, C. (2017). ioprospección de hongos micorrízicos arbusculares como alternativa para el fortalecimiento del cultivo de aguacate (Persea americana Miller) en Colombia. Revista de Investigación Agraria y Ambiental , 8(1), 1 - 10. Obtenido de 
http://hemeroteca.unad.edu.co/index.php/riaa/article/view/183 $9 / 2220$

Montenegro Gómez, S. P., \& Hernández Ossa, Y. K. (2015).

Biotecnología aplicada al desarrollo agropecuario colombiano.

Revista de Investigación Agraria y Ambiental, 6(2), 1 - 12.

Obtenido de

http://hemeroteca.unad.edu.co/index.php/riaa/article/view/140 $\underline{8 / 1732}$

Montes, J. y Sánchez, W. (2016). Relación Sociedad Civil, Estado y Economía en el mundo contemporáneo. Revista Criterio Libre Jurídico. 13(2), 36-49

http://dx.doi.org/10.18041/crilibjur.2016.v13n2.26203

Organización de las Naciones Unidas; 2012; Manual de Buenas

Prácticas Agrícolas, recuperado de

http://www.fao.org/home/es/

Toro Calderón, J., \& Martínez Prada, R. (2013). Métodos de Evaluación de Impacto Ambiental en Colombia. Revista de Investigación Agraria y Ambiental, 4(2), 43-53. doi:https://doi.org/10.22490/21456453.990

Vinasco Guzmán, M. (2017). Marco teórico para la construcción de una propuesta de turismo rural comunitario. Revista de Investigación Agraria y Ambiental, 8(1), 95 - 106. doi:https://doi.org/10.22490/21456453.1841

Zapata Galvis, J., \& Llano Franco, J. (2014). Perspectiva interdisciplinaria del ordenamiento alimentario y derecho del consumidor en Argentina. Revista de Investigación Agraria y Ambiental, 5(2), 99 - 114. doi:https://doi.org/10.22490/21456453.1329 